\title{
Artifacts for Agile User-Centered Design: A Systematic Mapping
}

\author{
Andrei Garcia \\ PUCRS, Faculdade de Informática \\ Porto Alegre, Brazil \\ Email: andrei.garcia@acad.pucrs.br
}

\author{
Tiago Silva da Silva \\ UNIFESP, Universidade Federal de São Paulo \\ São José dos Campos, Brazil \\ Email: silvadasilva@unifesp.br
}

\author{
Milene Selbach Silveira \\ PUCRS, Faculdade de Informática \\ Porto Alegre, Brazil \\ Email: milene.silveira@pucrs.br
}

\begin{abstract}
The integration of Agile and User-Centered Design methods is a fundamental condition to improve the quality of software products. However, one of the main problems faced to establish this integration on a day-to-day basis is how to improve communication among the invariably distinct involved teams. We believe that the artifacts involved in the development process could be used to support and increase teams' communication. To better understand this scenario, we performed a systematic mapping study on artifacts and their role in the communication between Agile and User-Centered Design fields. Through the analysis of 56 papers dealing with this specific topic, we present the artifacts used for communication in these approaches and software development events when they are used. The analyzed studies reinforced our beliefs about the importance of artifacts to improve teams' communication.
\end{abstract}

\section{INTRODUCTION}

Communication is crucial for the success of any business. The communication issue is one of the reasons why Software Engineering (SE) and Human-Computer Interaction (HCI) fields have been historically so distant from each other. Developers - with strong background in SE - and Designers - with background in HCI - use different vocabularies. The success of Agile Methods along with the popularization and increasing importance of User-Centered Design (UCD) in current products have reduced this distance.

Agile development has become mainstream regarding software development processes. Along with it, there is an increasing understanding of the importance of good user experiences. However, despite the fact that both aim to build software products with high quality, Agile methods and UCD methods approach development from a different perspective [1].

The integration of both fields is an essential requirement to increase the quality of software products. The overall picture of this integration is robust enough for researchers and practitioners [2]. However, such action on a day-to-day basis is still a concern, and one of its main problems is how to improve communication between developers and designers in order to build a shared understanding.

The collaboration between designers and developers is critical to the success of several agile projects, but little has been known about how this collaboration works [3]. We believe that this communication issue might be addressed by the use of artifacts as a communication mediator. Artifacts are a central means of communication for Agile and User-Centered Design software development [4].

Brhel et al. [4] identified five generic principles for the integration of Agile and User-Centered Design. The fifth principle is the "Artifact-Mediated Communication". According to the authors [4], an artifact is defined as an "... . aspect of the material world that has been modified over the history of its incorporation into goal-directed human action.". This principle consists of the use of tangible and up-to-date artifacts - accessible to all involved stakeholders - to document and communicate product and design concepts.

According to these authors, artifact-mediation communication has intensively been leveraged in other industries for many years. An example of such thing is clay modeling in the automotive industry when designing new cars. Surprisingly, empirical evidence for this important principle in the context of Agile UCD is too limited.

In order to provide a deeper understanding of contingency factors and their influence on design and development outcomes, the goal of this paper is to identify which artifacts are used in which context in order to facilitate the communication in an Agile User-Centered Design approach.

To achieve this goal, we carried out a systematic mapping study on the subject, extending a previous literature review about Agile and UCD [4]. In the mapping herein presented, besides the update of the search period - including 2015 and 2016/1 - we focused on the artifacts and their role in the communication between the fields of Agile and UCD. We found and analyzed 56 papers dealing with this specific subject.

In order to contextualize our findings, in the next section we present the background to understand our research: Agile, User-Centered Design and Agile User-Centered Design. In the Research Method we detail the protocol of our systematic mapping study. In Results we present the analysis of our results in order to answer our research questions. Finally, we present our conclusions and future work.

\section{BACKGROUND}

Next we will outline the theoretical background of Agile, User-Centered Design and Agile User-Centered Design. 


\section{A. Agile}

According to Larman [5], it is not possible to define Agile methods, as specific practices vary. However, these methods apply timeboxed iterative and evolutionary development, as well as adaptive planning, promoting evolutionary delivery, and including other values and practices that encourage agility - rapid and flexible response to change.

In addition, they promote practices and principles that reflect an agile sensibility of simplicity, lightness, communication, self-directed teams and programming over documenting, for instance.

In 2001, in Salt Lake City, a group interested in iterative and agile methods met to find common ground. The Agile Manifesto resulted from this meeting, which states that:

- Individuals and interactions over processes and tools.

- Working software over comprehensive documentation.

- Customer collaboration over contract negotiation.

- Responding to change over following a plan.

This generated the Agile principles that have guided the Agile development. These principles have also guided a number of Agile methods, e.g. Scrum [6], eXtreme Programming (XP) [7] and Crystal [8], just to name a few.

Among the existing Agile methods, Scrum or Scrum/XP hybrid approach dominates the Agile software industry with $70 \%$ of respondents of the [9].

\section{B. User-Centered Design}

Usability is the aspect of HCI devoted to ensuring that human-computer interaction is, among other things, effective, efficient, and satisfying for the user. Thus, usability includes characteristics such as ease of use, productivity, efficiency, effectiveness, learnability, retainability, and user satisfaction ISO9241.

User-Centered Design is the practice of focusing on the users. There is an international standard that is the basis for many UX/UCD methodologies. This standard (ISO 13407: Human-centred design process) [10] defines a general process for including human-centered (user-centered) activities throughout a development life-cycle, but does not specify exact methods.

In this model, once the need to use a human centered design process has been identified, four activities form the main cycle of work:

1) Specifying the context of use: Identifying the people who will use the product, what they will use it for, and under what conditions they will use it.

2) Specifying requirements: Identifying any business requirements or user goals that must be met for the product to be successful.

3) Creating design solutions: This part of the process may be done in stages, building from a rough concept to a complete design.

4) Evaluating designs: The most important part of this process is that evaluation - ideally through usability testing with actual users - is as integral as quality testing is to good software development.
The process ends - and the product can be released - once the requirements are met. However, in Agile contexts there is no end for the process.

\section{Agile User-Centered Design}

Agile UCD evolved from different motivations. On one hand, software engineers aim to satisfy customers through timely releases and responsiveness to change requests without compromising software quality. On the other hand, UCD aims at ensuring appropriate usability of the implemented software, a characteristic that has not been sufficiently considered in traditional, plan-driven approaches or in agile approaches. UCD addresses this issue but does not consider Agile principles [4].

First attempts to integrate Agile and UCD approaches were made about a decade ago, and as already mentioned, the overall picture of this integration is firm enough. For instance, Sy [11], Ferreira et al. [12], Fox et al. [13], and Silva et al. [14] came up with very similar proposals.

Salah, Paige and Cairns [15] performed a systematic review to identify restriction factors regarding Agile and UCD integration, and explored practices to deal with them. One of their findings in this review was about the dynamics between developers and designers which talks about the ongoing and continuous communication between the teams. Regarding sharing and understanding design tasks, a number of practices are used, such as design studio, developers participating in UI specifications and shared artifacts within the team.

Therefore, in order to better understand this integration on a day-to-day basis and believing that the artifact-mediated communication is a way for building a shared understanding between designers and developers, we carried out a systematic mapping about Agile UCD artifacts.

\section{Research Method}

A Systematic Mapping Study is a method designed to provide an overview of a research field by verifying the research data existence and by providing the amount and classification of such research data [16]. This study was performed following the guidelines for conducting systematic mapping studies described by Petersen [17]. According to the author, the mapping process consists of planning, conducting and reporting. Next sub-sections provide details on how each phase was performed from planning to conducting the mapping.

\section{A. Planning}

Before conducting the mapping, we planned in details to define the search question and establish the research protocol. The protocol was defined bearing in mind the steps of search strategy, selection criteria, and data extraction strategy. As stated by Kitchenham [16], a protocol is necessary to reduce chances of researcher bias.

1) Research Question: The main goal of this mapping is to identify the artifacts that may improve communication in an Agile User-Centered Design approach. Therefore, we defined three primary research questions: 
- RQ1: Which are the artifacts that facilitate communication between Agile Methods and User-Centered Design areas?

- RQ2: Which event of the process are these artifacts being used?

- RQ3: Are these artifacts physical or electronic?

By answering these research questions, this systematic mapping provides an overview about the artifacts used in Agile User-Centered Design process that facilitate team communication. Moreover, this mapping shall identify research gaps and trends with regards to this topic.

2) Search Strategy: As described in the background section, there are some relevant systematic reviews about Agile Methods and UCD integration, for instance, [14], [15], [4]. The most recent is the systematic review performed by Brhel et al. [4]. Since their study captured the state of Agile and UCD integration, we decided to replicate part of their search strategy. However, our study focuses on mapping the artifacts that might mediate the communication within this context.

The computer science literature, including the HCI subdomain is available in six relevant databases, namely ACM Digital Library ${ }^{1}$, EBSCO Host $^{2}$, Elsevier ScienceDirect ${ }^{3}$, IEEE Xplore $^{4}$, ProQuest ${ }^{5}$, and Springer Link ${ }^{6}$. All of them were used to search for primary studies.

In order to automate the search in the selected databases, a search string was composed using keywords from both UserCentered Design and Agile Methods. Almost all keywords from Brhel et al. [4] study were used, except for "software development" and "systems development", which were removed from our search string in order to broaden the first results. Table I shows the search string and its variations according to each database. For the Elsevier's database, it was necessary to specify the search on title, keywords and abstract fields. To run the search string within the Springer's database, we consulted the authors from [4] to understand how they used it within this database. Due to Springer's search field limitation of number of words, the search string had to be divided into three parts.

The timeframe defined for our systematic mapping included studies published from 2002 to 2016. This starting date was selected because Agile Manifesto arose in 2001 and the publication of papers related to this field has started in 2002.

3) Selection Criteria: We evaluated each publication retrieved from the automated search in order to select whether or not it should be included by considering titles and abstracts. In a first filter, we excluded papers based only on titles and abstracts. In a second filter, we ensured a full text reading.

The following inclusion criteria were applied in the first filter:

- Studies should be published in the computer science area.

\footnotetext{
${ }^{1}$ http://portal.acm.org

${ }^{2}$ https://www.ebscohost.com/

${ }^{3} \mathrm{http}: / / \mathrm{www}$. sciencedirect.com

${ }^{4} \mathrm{http}: / / \mathrm{www}$.ieeexplore.ieee.org/Xplore

${ }^{5} \mathrm{http}: / /$ www.proquest.com/

${ }^{6} \mathrm{http}: / /$ link.springer.com/
}

TABLE I

SEARCH StRING VARIATIONS ACCORDING TO EACH DATABASE

\begin{tabular}{|c|c|}
\hline Database & Search String \\
\hline $\begin{array}{l}\text { ACM } \\
\text { EBSCO } \\
\text { IEEE } \\
\text { ProQuest }\end{array}$ & $\begin{array}{l}\text { (ergonomics OR "human-computer interaction" } \\
\text { OR "computer-human interaction" OR "interaction design" } \\
\text { OR usability OR "user experience" OR "user-centered } \\
\text { design" OR "ui design" OR "interface design") AND } \\
\text { (agile OR scrum OR "extreme programming" OR lean OR } \\
\text { "crystal clear" OR "feature driven development" OR "dynamic } \\
\text { software development") }\end{array}$ \\
\hline Elsevier & $\begin{array}{l}\text { Search String: ((ergonomics OR "human-computer } \\
\text { interaction" OR "computer-human interaction" OR } \\
\text { "interaction design" OR usability OR "user experience" OR } \\
\text { "user-centered design" OR "ui design" OR "interface } \\
\text { design") AND (agile OR scrum OR "extreme programming" } \\
\text { OR lean OR "crystal clear" OR "feature driven development" } \\
\text { OR "dynamic software development")) } \\
\text { (TITLE(Search String)) OR (KEYWORDS(Search String)) } \\
\text { OR (ABSTRACT(Search String)) }\end{array}$ \\
\hline Springer & $\begin{array}{l}\text { Search String } 1 \text { (Agile OR } \\
\text { Scrum OR "Extreme Programming"): } \\
\text { ab((ergonomics OR "human-computer } \\
\text { interaction" OR "interaction design" OR usability OR } \\
\text { "user experience" OR "user-centered design") AND (agile } \\
\text { OR scrum OR "extreme programming")) } \\
\text { Search String } 2 \text { (Lean OR } \\
\text { "Crystal Clear"): } \\
\text { ab((ergonomics or "human-computer } \\
\text { interaction" or "interaction design" or usability or } \\
\text { "user experience" or "user-centered design") and (Lean OR } \\
\text { "Crystal Clear")) } \\
\text { Search String } 3 \\
\text { ("Feature Driven Development" OR "Dynamic Software } \\
\text { Development"): } \\
\text { ab((ergonomics or "human-computer } \\
\text { interaction" or "interaction design" or usability or } \\
\text { "user experience" or "user-centered design") and } \\
\text { ("Feature Driven Development" OR "Dynamic Software } \\
\text { Development")) }\end{array}$ \\
\hline
\end{tabular}

- Studies should present the subject on Agile Methods and UCD integration.

Publications that met at least one of the following exclusion criteria were removed:

- Books.

- Duplicated papers.

- Studies written in any language other than English.

- Studies presenting summaries of tutorials, panels, poster sessions or workshops.

- Conference covers and table of content.

During the full text reading stage, we performed a more detailed analysis of the paper content. The goal of this stage was to select the studies according the following inclusion criteria:

- Studies should present artifacts used in Agile UserCentered Design.

- Studies should present artifacts that facilitate team communication (e.g. Communication between developer and designer).

Studies that were referring to some artifacts, but not regarding communication between teams or people, were excluded. 


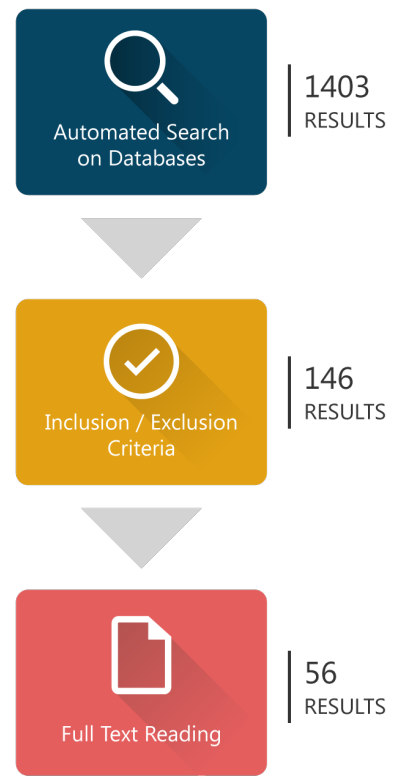

Fig. 1. Selection process

TABLE II

POSSIBLE ANSWERS TO DEFINE A DATA EXTRACTION STRATEGY

\begin{tabular}{ll}
\hline Research Question & Possible Answers \\
\hline & Sketch \\
& Persona \\
RQ1: Which are the artifacts that & Mockup \\
facilitate communication & Scenario \\
between Agile Methods and & Guideline \\
User-Centered Design areas? & Prototype \\
& User Story \\
& Storyboard \\
RQ2: Which event of the process & Discovery \\
are these artifacts being used? & Iterative Cycle \\
\hline RQ3: Are these artifacts physical & Physical \\
or electronic? & Electronic \\
\hline
\end{tabular}

4) Data Extraction Strategy: The data extraction strategy was based on providing a set of possible answers for the research questions. Since RQ1 and RQ2 could retrieve a great number of answers, we defined some initial possible answers. This set of possible answers was extended throughout screening.

Regarding RQ1, we created a list of possible artifacts that facilitate communication on Agile UCD. The same strategy was defined for RQ2 and RQ3. With regards to RQ2, we created a list of possible events in which the artifacts were used. For RQ3 there were only two possible answers. Table II displays the set of initial possible answers for each question.

\section{B. Conduction}

We searched for papers in the selected databases during April 2016. The first results led us to a set of 1403 studies (Table III). After the results' compilation, we applied the exclusion criteria, resulting in 1200 publications. Afterwards,
TABLE III

NUMBER OF PUBLICATIONS PER DATABASE

\begin{tabular}{l|c|c|c}
\hline Data Base & Search & First Filter & Final set \\
\hline ACM & 306 & 57 & 18 \\
EBSCO & 176 & 2 & 0 \\
Elsevier & 14 & 5 & 3 \\
IEEE & 226 & 54 & 29 \\
ProQuest & 129 & 10 & 4 \\
Springer & 552 & 18 & 2 \\
\hline
\end{tabular}

TABLE IV

SELECTED PAPERS

\begin{tabular}{ll}
\hline Year & References \\
\hline 2002 & {$[18][19]$} \\
2003 & {$[20]$} \\
2004 & {$[21]$} \\
2005 & {$[22][23]$} \\
2006 & {$[24][25][26]$} \\
2007 & {$[27][28][11][29]$} \\
2008 & {$[30][31][13][32][33][34][35][36][37]$} \\
2009 & {$[38][39][40][41]$} \\
2010 & - \\
2011 & {$[3][42][14]$} \\
2012 & {$[43][44][45][46][47]$} \\
2013 & {$[48][49][50][51][52]$} \\
2014 & {$[53][54][55][56][57][58]$} \\
2015 & {$[59][60][4][2][61][62][63][64][64][65]$} \\
2016 & {$[66]$} \\
\hline
\end{tabular}

a total of 146 papers were selected in accordance with the inclusion criteria from the first stage, where only the title and abstract were considered. Finally, in the full text reading stage, 56 publications were selected (Table IV). The selection process is shown in Figure 1.

\section{RESULTS}

The following subsections present the analysis of the results for each research question.

\section{A. Artifacts on Agile User-Centered Design}

The results for question RQ1 - Which are the artifacts that facilitate communication between Agile Methods and UserCentered Design fields? - revealed that there are at least 20 artifacts used to promote communication in Agile UserCentered Design. During data extraction, it becomes clear that our initial set of possible answers was not covering all the artifacts. Over the full text reading, we identified many artifacts that were included in possible answers to increment the data extraction definition. Additionally, we could realize that authors use different nomenclature for artifacts that are used for the same purpose. For example, the term guideline [64] [65] was also used as style guide [49], and design specification [60]. To handle these different names, we grouped the artifacts according to their objectives. The most common name and most cited terminology was used to group them. Table 
TABLE V

RESULTED ARTIFACTS

\begin{tabular}{|c|c|c|c|}
\hline Artifact Group & Artifact Used Name & Results & Total \\
\hline Blueprint & Blueprint & 1 & 1 \\
\hline Card & $\begin{array}{l}\text { Design Card } \\
\text { Feature Card } \\
\text { Issue Card } \\
\text { Task-Case Card } \\
\text { User Role Card }\end{array}$ & $\begin{array}{l}4 \\
4 \\
3 \\
3 \\
2\end{array}$ & 16 \\
\hline Guideline & $\begin{array}{l}\text { Guideline } \\
\text { Style Guide } \\
\text { Design Specification }\end{array}$ & $\begin{array}{l}2 \\
1 \\
1\end{array}$ & 4 \\
\hline List & List (Group of Ideas) & 1 & 1 \\
\hline Map & $\begin{array}{l}\text { Claim Map } \\
\text { Concept Map } \\
\text { Content Mapping } \\
\text { Effect Map } \\
\text { Site Map }\end{array}$ & $\begin{array}{l}3 \\
1 \\
1 \\
1 \\
1\end{array}$ & 7 \\
\hline Mockup & $\begin{array}{l}\text { HTML Mockup } \\
\text { Mockup } \\
\text { Paper Mockup }\end{array}$ & $\begin{array}{l}1 \\
6 \\
1\end{array}$ & 8 \\
\hline Model & $\begin{array}{l}\text { Content Model } \\
\text { Context Model }\end{array}$ & $\begin{array}{l}1 \\
1\end{array}$ & 2 \\
\hline Persona & $\begin{array}{l}\text { Adhoc Persona } \\
\text { Extreme Persona } \\
\text { Persona }\end{array}$ & $\begin{array}{l}1 \\
1 \\
13\end{array}$ & 15 \\
\hline Prototype & $\begin{array}{l}\text { Prototype } \\
\text { UI Prototype }\end{array}$ & $\begin{array}{l}28 \\
6\end{array}$ & 34 \\
\hline Research Results & Research Results & 1 & 1 \\
\hline Scenario & Scenarios & 12 & 12 \\
\hline Sketch & Sketches & 13 & 13 \\
\hline Storyboard & Storyboards & 5 & 5 \\
\hline Storytelling & $\begin{array}{l}\text { Oral Storytelling } \\
\text { Storytelling }\end{array}$ & $\begin{array}{l}1 \\
2\end{array}$ & 3 \\
\hline UI Design & $\begin{array}{l}\text { UI Design } \\
\text { UI Proposal } \\
\text { UI Screen } \\
\text { Visual Design }\end{array}$ & $\begin{array}{l}5 \\
1 \\
1 \\
1\end{array}$ & 8 \\
\hline Use Case & Use Case & 2 & 2 \\
\hline User Flow & $\begin{array}{l}\text { Flow Model } \\
\text { Task Model } \\
\text { User Flow } \\
\text { User Jorney }\end{array}$ & $\begin{array}{l}1 \\
2 \\
1 \\
2\end{array}$ & 6 \\
\hline User Story & $\begin{array}{l}\text { Card (User Story) } \\
\text { Stories } \\
\text { User Objective } \\
\text { User Story }\end{array}$ & $\begin{array}{l}2 \\
8 \\
2 \\
10\end{array}$ & 22 \\
\hline UX Target & UX Target & 1 & 1 \\
\hline Wireframe & Wireframe & 8 & 8 \\
\hline
\end{tabular}

$\mathrm{V}$ presents all the extracted artifacts, groups and extracted numbers for all selected papers. Figure 3 shows the list of extracted artifacts.

Next, we present some of the studies that highlight the importance of artifacts as an important channel of communication between different areas [23]. Beyer, Holtzblatt and Baker [21] state that, by using conceptual diagrams and mockups, team communication can be facilitated. In their study, task models were used to show the scope of issues from all customers to the entire team. After that, product marketing, development leads and human factors participated determining which issues would be addressed by the project. User stories were described according to sequence models. These user stories were used during planning meeting to define what would integrate an iteration. During Planning, high level mockups were used to facilitate team communication.

In her study, Tay [23] describes that a UI Prototype developed by software developers was effective in encouraging group communication. The UI Prototype evolved from storyboards. Testers used storyboards to test the workflow.

Meszaros and Aston [67] took advantage from paper prototypes to run usability tests and, after that, used these prototypes as a UI storyboard, where they taped up all story cards. This storyboard became an important means of communication during the Planning Meeting.

In their project, Patton [22] described that they used task cards to describe user tasks during the span plan. This user tasks were used as base to build low fidelity paper prototypes. Whit the prototypes supporting the most important tasks they could write the user stories. With all these artifacts, especially the prototypes, developers could estimate and build software more accurately.

According to results, prototypes seem to be the most used artifact to facilitate communication on Agile User-Centered Design. In addition to that, brief documentation and face-toface communication can be used to integrate the team [68].

\section{B. Events in which artifacts are used}

The results for RQ2 - Which event of the process are these artifacts being used? - revealed at least 5 events involving artifacts as means of communication between team members. Our initial set of possible answers for events included Discovery, Iterative Cycle and Planning Meeting. However, throughout data extraction two more events were included, namely Review Meeting and General Meetings. Resulted events were grouped according to their purposes and are displayed in Table VI.

Planning event appears in 17 studies. Pre-Planning [27], [38], Pre Release Planning [27], Span Plan [22], and Plan Parallel Iteration [46] were also considered as Planning since their main goal was to define what would integrate an iteration. For the planning period, UI design and user stories are delivered to the development team and they can use them to estimate how much effort they need for the next iteration [21]. Cards are the most used artifact on planning event, as seen in Figure 2. During an iteration planning, cards are used to estimate work and, during this process, the designer gives more details to the engineering manager and developers to have a more accurate estimation and to figure out what is going to be done in the iteration [27].

Iterative Cycle is a process based on iterative and incremental cycles of software production [52], which includes both development and design tasks. During this iterative stage, many artifacts are used to mediate communication, mainly 
EVENTS

\begin{tabular}{|c|c|c|c|c|c|}
\hline & Discovery & Planning & Iterative Cycle & Review Meeting & General Meetings \\
\hline BLUEPRINT & 0 & 1 & 0 & 0 & 0 \\
\hline CARDS & 4 & 5 & 7 & 1 & 0 \\
\hline GUIDELINES & 0 & 0 & 0 & 0 & 0 \\
\hline LIST & 0 & 0 & 0 & 0 & 0 \\
\hline MAPS & 0 & 0 & 3 & 1 & 0 \\
\hline MOCKUP & 0 & 0 & 4 & 1 & 0 \\
\hline MODELS & 0 & 0 & 0 & 2 & 0 \\
\hline PERSONA & 0 & 2 & 3 & 0 & 1 \\
\hline PROTOTYPE & 0 & 3 & 15 & 0 & 3 \\
\hline RESEARCH RESULTS & 0 & 0 & 1 & 0 & 0 \\
\hline SCENARIO & 1 & 1 & 3 & 0 & 0 \\
\hline SKETCH & 1 & 0 & 0 & 0 & 1 \\
\hline STORYBOARD & 1 & 1 & 0 & 0 & 0 \\
\hline STORYTELLING & 0 & 0 & 3 & 0 & 0 \\
\hline UI DESIGN & 2 & 1 & 5 & 0 & 0 \\
\hline USE CASE & 0 & 0 & 0 & 0 & 0 \\
\hline USER FLOW & 1 & 0 & 1 & 0 & 0 \\
\hline USER STORY & 0 & 4 & 9 & 0 & 0 \\
\hline UX TARGET & 0 & 1 & 0 & 0 & 0 \\
\hline WIREFRAME & 0 & 1 & 3 & 0 & 0 \\
\hline
\end{tabular}

Fig. 2. Artifacts $X$ Events

between developers and designers. As shown in Figure 2, the most used artifact on this event is prototype, followed by user story and cards. 31 studies reported the use of some artifact during this event. Sy [11] explains that in this event the UI Design is presented in person to the developers who will implement it. A typical workflow is demonstrated on the last available prototype.

Five studies [69][45][43][56][3] show the use of artifacts in meetings with no specific subject. We called them as General Meetings, since we did not know the exact meetings' topic. Basically, prototypes, personas and sketches are used during these meetings to facilitate communication. Interactive prototypes and whiteboard sketches are examples of interface proxies between designers and developers [3].

\section{Artifacts Format}

Considering studies that describe artifacts' format, the results for RQ3 - Are these artifacts physical or electronic? expose that $48 \%$ of artifacts are available in physical format such as sketches and paper prototypes. On the other hand, 52\% of the artifacts are available in electronic format, as in Table VII.

Paper and whiteboards are used as tools to create physical artifacts. Most common examples of paper artifacts are Cards, Sketches and Paper Prototypes. In addition, these type of artifacts are commonly used throughout planning and discovery events. Paper prototypes are posted as a user interface story-
TABLE VI

RESULTED EVENTS

\begin{tabular}{|c|c|c|c|}
\hline Event Group & Event Name & Results & Total \\
\hline \multirow{5}{*}{ Discovery } & Build an Affinity & 1 & \multirow{5}{*}{7} \\
\hline & Design Studio & 1 & \\
\hline & Initial Workshop & 2 & \\
\hline & U-CD Session & 2 & \\
\hline & UX Design Phase & 1 & \\
\hline \multirow{5}{*}{ Planning } & Plan Parallel Iteration & 1 & \multirow{5}{*}{17} \\
\hline & Planning & 13 & \\
\hline & Pre-Planning & 1 & \\
\hline & Pre Release Planning & 1 & \\
\hline & Span Plan & 1 & \\
\hline \multirow{5}{*}{ Iterative Cycle } & During Development Cycle & 12 & \multirow{5}{*}{31} \\
\hline & Iterative Stage & 5 & \\
\hline & Iterative UI Design & 1 & \\
\hline & Next Sprint & 9 & \\
\hline & One Sprint Ahead & 4 & \\
\hline \multirow{2}{*}{ Review Meeting } & Review Meeting & 1 & \multirow{2}{*}{2} \\
\hline & Product Backlog Manag & 1 & \\
\hline General Meetings & Meetings & 5 & 5 \\
\hline
\end{tabular}

board in planning meetings to facilitate team communication [67]. Paper mockups are also used to collect and present ideas between developers and designers [70].

Regarding electronic artifacts, they are available using technologies such as Central Design Record, Power Point, Balsamiq, Shockwave, and HTML. It is important to emphasize 


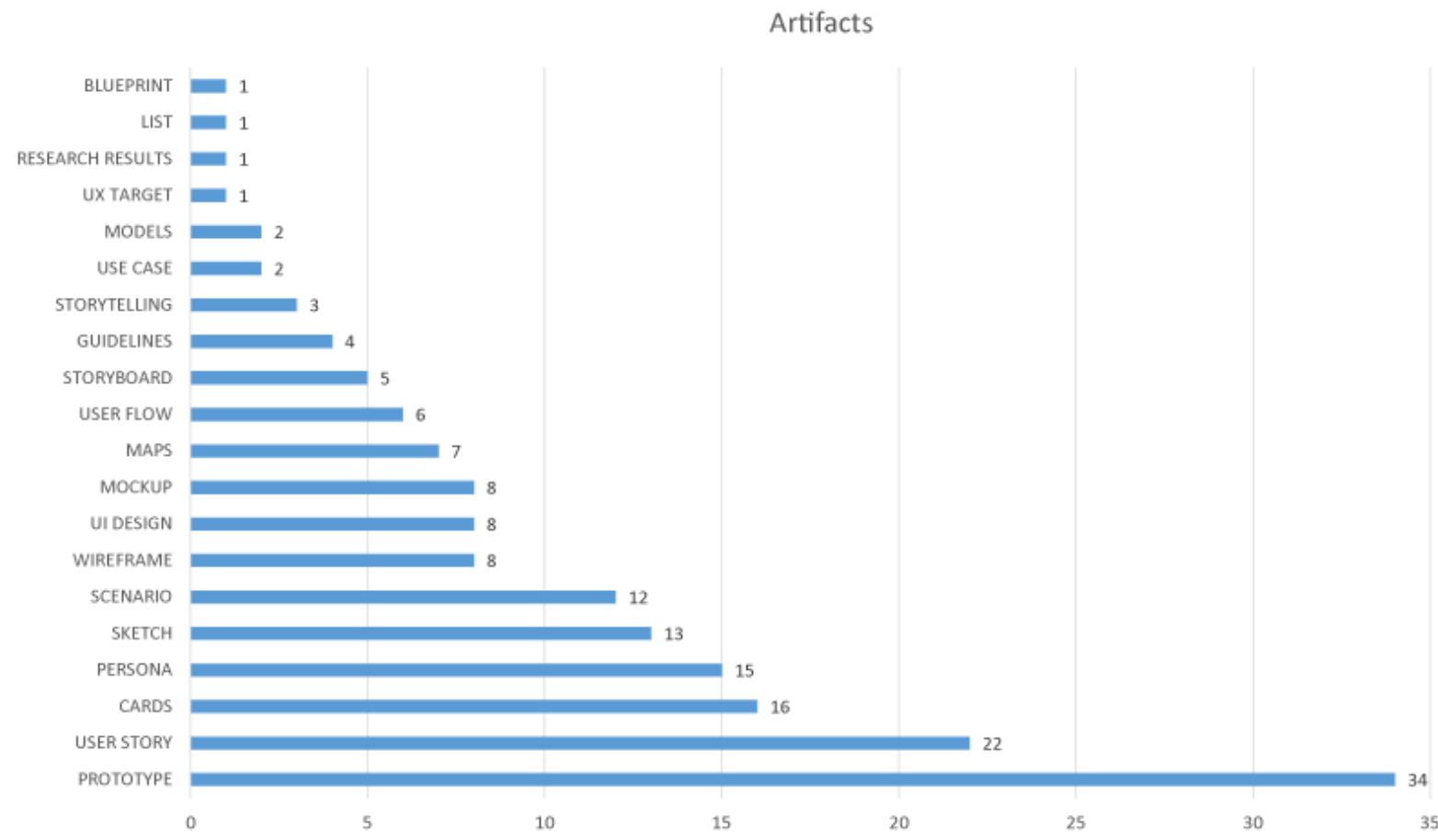

Fig. 3. Artifacts used to facilitate communication

TABLE VII

RELATION BETWEEN ARTIFACTS' FORMAT AND EVENTS

\begin{tabular}{lllll}
\hline Artifact Format & Event & Per Event & Total & \% \\
\hline \multirow{4}{*}{ Physical } & Discovery & 6 & & \\
& Planning & 7 & & \\
& Iterative Cycle & 7 & 30 & $48 \%$ \\
& Review Meeting & 1 & & \\
& General Meeting & 1 & & \\
& Data Not Available & 8 & & \\
Electronic & Discovery & 2 & 33 & $52 \%$ \\
& Planning & 1 & & \\
\hline & Iterative Cycle & 17 & &
\end{tabular}

that electronic artifacts are mostly used during the iterative cycle. This event requires more detailed artifacts, since at this point they often serve as basis for development. For instance, "paper mock-ups are used to get the basic concepts right while HTML mock-ups are used for a more detailed view" [70].

\section{Discussion}

Many artifacts appear as facilitators in communication between Agile and UCD, and this mapping study presented 20 different artifact groups playing this important role (Table V). Prototypes and User Story show up as the most used artifacts. Besides that, these artifacts are used in different events during the agile development process, such as planning and iterative cycle. Table VI shows the five event groups where these artifacts benefit discussion between teams.
Prototypes are mostly used during iterative cycle, as shown in Figure 2. This happens due to the fact that prototypes can address different goals, from serving as reference for development until acting as an instrument for usability tests. This visual representation can provide details for the development team and it is used as a vehicle to trigger discussions. For instance, Lievesley and Yee [25] described in their case study that prototypes were posted in the developers' team workspace as a regular point of reference.

Prototypes also seem to be relevant during planning meetings, as well as user stories and cards, where they are used to define what will integrate an iteration. During the estimation process, designers use these artifacts to give more details to the engineer team for a more accurate estimation [27]. In general at this event, artifacts use to be more flexible and lightweight to facilitate face-to-face communication.

Physical artifacts are more used in events that required more face-to-face communication, such as planning and discovery. Generally speaking, they are used as base to generate other artifacts, for instance, prototypes and cards serving as base to create User Stories [27]. On the other hand, electronic artifacts are used in events that require more details, and can serve as acceptance criteria and guideline for a User Story. Whether physical or electronic, artifacts seem to be an important channel of communication between different domains.

\section{CONCLUSION}

Both Agile and UCD methods intend to build quality software under different perspectives. Agile UCD approaches 
try to close the gaps between these two areas, bringing to the software development process the most effective techniques, methods, and artifacts of each of them. However, not only do different perspectives affect this integration; but also communication between different professional profiles - at least designers and developers - have a high impact on it.

In the research herein presented, we focused on the artifacts used to promote communication in Agile UCD approach. Through a mapping study about integration of both areas, we deepen our understanding not only about the artifacts used for communication in these approaches, but also the software development events they are used. Once there is no consensus concerning the terms used both to artifacts and events of the software development process, we shall categorize the results found in order to better understand, reflect on and present them.

The mapping study presented a total of 20 artifact groups related to the analyzed research papers. Regarding these groups, Prototypes, User Stories, Cards, and Personas are the most cited ones (with 34, 22, 16, and 15 citations, respectively). Considering development events, the studies revealed 5 distinct events involving artifacts used as communication means. The Iterative Cycle was the event with the greatest sharing of artifacts, and, in this event, Prototypes (15), User Stories (9), and Cards (7), once again were the most cited artifacts. Finally, artifacts are found both in physical and electronic formats. Physical artifacts are commonly used throughout planning and discovery events; whereas the electronic ones are mostly used during the iterative cycle, often being used as basis for development.

The analyzed studies show us the importance of artifacts to increase teams' communication. Now we intend to extend our understanding, investigating the teams' perspective about the artifacts' role in communication improvement through a series of interviews with different professionals who work in Agile UCD teams. Furthermore, it is possible to research on the impact of different artifacts' combination. Another perspective to be studied is the communication not only between development and design teams, but also extended to reach the strategic levels of decision-making.

\section{ACKNOWLEDGMENT}

The authors would like to thank FAPESP.

\section{REFERENCES}

[1] J. Ferreira, H. Sharp, and H. Robinson, "User experience design and agile development: managing cooperation through articulation work," Softw. Pract. Exper, vol. 41, pp. 963-974, August 2011.

[2] T. S. Da Silva, M. S. Silveira, and F. Maurer, "Usability evaluation practices within agile development," Proceedings of the Annual Hawail International Conference on System Sciences, vol. 2015-March, pp. 5133-5142, 2015.

[3] J. M. Brown, G. Lindgaard, and R. Biddle, "Collaborative events and shared artefacts: Agile interaction designers and developers working toward common aims," Proceedings - 2011 Agile Conference, Agile 2011, pp. 87-96, 2011.

[4] M. Brhel, H. Meth, A. Maedche, and K. Werder, "Exploring principles of user-centered agile software development: A literature review." Information \& Software Technology, vol. 61, pp. 163-181, may 2015.
[5] C. Larman, Agile and Iterative Development: A Manager's Guide. Pearson Education, 2003.

[6] K. Schwaber and M. Beedle, Agile Software Development with Scrum, 1st ed. Upper Saddle River, NJ, USA: Prentice Hall PTR, 2001.

[7] K. Beck and C. Andres, Extreme Programming Explained: Embrace Change (2Nd Edition). Addison-Wesley Professional, 2004.

[8] A. Cockburn, Crystal Clear a Human-powered Methodology for Small Teams, 1st ed. Addison-Wesley Professional, 2004.

[9] V. One, "10th annual state of agile report," Version One, Tech. Rep., 2016.

[10] ISO13407, "ISO 13407:1999, Human-centred design processes for interactive systems," International Standards Organization, Tech. Rep., 2011.

[11] D. Sy, "Adapting Usability Investigations for Agile User-Centered Design,” Journal of Usability Studies, vol. 2, no. 3, pp. 112-132, 2007.

[12] J. Ferreira, H. Sharp, and H. Robinson, "Values and assumptions shaping agile development and user experience design in practice," in $X P, 2010$, pp. 178-183.

[13] D. Fox, J. Sillito, and F. Maurer, "Agile Methods and User-Centered Design: How These Two Methodologies are Being Successfully Integrated in Industry," Agile 2008 Conference, pp. 63-72, 2008.

[14] T. S. Da Silva, A. Martin, F. Maurer, and M. Silveira, "User-centered design and agile methods: A systematic review," Proceedings - 2011 Agile Conference, Agile 2011, pp. 77-86, 2011.

[15] D. Salah, R. F. Paige, and P. Cairns, "A systematic literature review for agile development processes and user centred design integration," in Proceedings of the 18th International Conference on Evaluation and Assessment in Software Engineering, ser. EASE '14. New York, NY, USA: ACM, 2014, pp. 5:1-5:10. [Online]. Available: http://doi.acm.org/10.1145/2601248.2601276

[16] B. Kitchenham and S. Charters, "Guidelines for performing Systematic Literature Reviews in Software Engineering," Keele University and Durham University Joint Report, Tech. Rep. EBSE 2007-001, 2007.

[17] K. Petersen, R. Feldt, S. Mujtaba, and M. Mattsson, "Systematic mapping studies in software engineering," in Proceedings of the 12th International Conference on Evaluation and Assessment in Software Engineering, ser. EASE'08. Swinton, UK, UK: British Computer Society, 2008, pp. 68-77. [Online]. Available: http://dl.acm.org/citation.cfm?id=2227115.2227123

[18] L. L. Constantine and L. A. D. Lockwood, "Usage-centered engineering for web applications," IEEE Software, vol. 19, no. 2, pp. 42-50, 2002.

[19] J. Patton, "Hitting the Target: Adding Interaction Design to Agile Software Development," 2002.

[20] J. Hakim, T. Spitzer, and J. Armitage, "Sprint: Agile specifications in Shockwave and Flash," Proceedings of the 2003 conference on ..., pp. 1-14, 2003. [Online]. Available: http://dl.acm.org/citation.cfm?id=997111

[21] H. Beyer, K. Holtzblatt, and L. Baker, "An Agile Customer-Centered Method : Rapid Contextual Design," Extreme Programming and Agile Methods-XP/Agile Universe 2004, no. Cd, pp. 50-59, 2004.

[22] J. Patton, "Finding the forest in the trees," in Companion to the 20th annual ACM SIGPLAN conference on Object-oriented programming, systems, languages, and applications, ser. OOPSLA '05. New York, NY, USA: ACM, 2005, pp. 266-274.

[23] G. Tai, "A communication architecture from rapid prototyping," $A C M$ SIGSOFT Software Engineering Notes, vol. 30, no. 4, p. 1, 2005.

[24] J. Honious and J. Clark, "Something to Believe In," Agile 2006 (Agile'06), pp. 203-212, 2006. [Online]. Available: http://ieeexplore.ieee.org/lpdocs/epic03/wrapper.htm?arnumber=1667581

[25] M. A. Lievesley and J. S. R. Yee, "The role of the interaction designer in an agile software development process," $\mathrm{CHI}$ '06 extended abstracts on Human factors in computing systems - CHI EA 'O6, p. 1025, 2006. [Online]. Available: http://www.scopus.com/inward/record.url?eid=2s2.0-84868273593\&partnerID=tZOtx3y 1

[26] G. Meszaro and J. Aston, "Adding usability testing to an agile project," Proceedings - AGILE Conference, 2006, vol. 2006, pp. 289-294, 2006.

[27] J. Ferreira, J. Noble, and R. Biddle, "Agile development iterations and UI design," Proceedings - AGILE 2007, pp. 50-58, 2007.

[28] J. C. Lee, D. S. Mccrickard, and V. Tech, "Towards Extreme ( ly ) Usable Software : Exploring Tensions Between Usability and Agile Software Development," Agile Conference (AGILE), 2007, 2007. [Online]. Available: http://ieeexplore.ieee.org/xpls/abs_all.jsp?arnumber $=4293576$

[29] H. Williams and A. Ferguson, "The ucd perspective: Before and after agile," in AGILE 2007, 2007, pp. $285-290$. 
[30] D. Broschinsky and L. Baker, "Using persona with XP at LANDesk software, an avocent company," Proceedings - Agile 2008 Conference, pp. 543-548, 2008.

[31] J. Brown, G. Lindgaard, and R. Biddle, "Stories, sketches, and lists: Developers and interaction designers interacting through artefacts," Proceedings - Agile 2008 Conference, pp. 39-50, 2008.

[32] M. Najafi and L. Toyoshiba, "Two Case Studies of User Experience Design and Agile Development," Agile 2008 Conference, pp. 531-536, 2008.

[33] Z. H. Sain, M. Lechner, H. Milchrahm, S. Shahzad, W. Slaný, M. Umgeher, T. Vlk, and P. Wolkerstorfer, "User interface design for a mobile multimedia application: An iterative approach," Proceedings of the 1st International Conference on Advances in Computer-Human Interaction, ACHI 2008, pp. 189-194, 2008.

[34] M. Singh, "U-SCRUM: An agile methodology for promoting usability," Proceedings - Agile 2008 Conference, pp. 555-560, 2008. [Online]. Available: http://ieeexplore.ieee.org/lpdocs/epic03/wrapper.htm?arnumber=4599538

[35] J. Ungar and J. White, "Agile User Centered Design: Enter the Design Studio - A Case Study," Proceeding of the twenty-sixth annual CHI conference extended abstracts on Human factors in computing systems - CHI '08, p. 2167, 2008.

[36] J. Ungar, "The design studio: Interface design for agile teams," Proceedings - Agile 2008 Conference, pp. 519-524, 2008.

[37] P. Wolkerstorfer, M. Tscheligi, R. Sefelin, H. Milchrahm, Z. Hussain, M. Lechner, and S. Shahzad, "Probing an agile usability process," Proceedings of ACM CHI 2008 Conference on Human Factors in Computing Systems, vol. 2, pp. 2151-2158, 2008. [Online]. Available: http://doi.acm.org/10.1145/1358628.1358648

[38] M. Budwig, S. Jeong, and K. Kelkar, "When user experience met agile: a case study," Proceedings of the 27th international conference extended abstracts on Human factors in computing systems, pp. 3075-3084, 2009.

[39] L. Cho, "Adopting an agile culture," Proceedings - 2009 Agile Conference, AGILE 2009, pp. 400-403, 2009.

[40] J. Kollmann, H. Sharp, and A. Blandford, "The importance of identity and vision to user experience designers on agile projects," Proceedings - 2009 Agile Conference, AGILE 2009, pp. 11-18, 2009.

[41] J. C. Lee, D. S. McCrickard, and K. T. Stevens, "Examining the foundations of agile usability with extreme scenario-based design," Proceedings - 2009 Agile Conference, AGILE 2009, pp. 3-10, 2009.

[42] J. C. Lee, T. K. Judge, and D. S. McCrickard, "Evaluating eXtreme scenario-based design in a distributed agile team," CHI '11 Extended Abstracts on Human Factors in Computing Systems, pp. 863-877, 2011. [Online]. Available: http://portal.acm.org/citation.cfm?doid=1979742.1979681

[43] J. M. Brown, G. Lindgaard, and R. Biddle, "Joint implicit alignment work of interaction designers and software developers," Proceedings of the 7th Nordic Conference on Human-Computer Interaction Making Sense Through Design - NordiCHI '12, p. 693, 2012.

[44] M. Isomursu, A. Sirotkin, P. Voltti, and M. Halonen, "User experience design goes agile in lean transformation - A case study," Proceedings 2012 Agile Conference, Agile 2012, pp. 1-10, 2012.

[45] K. Koerner, D. Dailey, M. Lipp, H. Connor, and R. Sharma, "Data visualization for psychotherapy progress tracking," Proceedings of the 30th ACM international conference on Design of communication SIGDOC '12, p. 213, 2012.

[46] B. Losada, M. Urretavizcaya, J.-M. López-Gil, and I. Fernández-Castro, "Combining InterMod agile methodology with usability engineering in a mobile application development," Proceedings of the 13th International Conference on Interacción Persona-Ordenador - INTERACCION '12, no. August, pp. 1-8, 2012.

[47] T. Silva and M. Silveira, "User experience design and agile development: From theory to practice," Journal of Software ..., vol. 05, no. 10, pp. 743-751, 2012.

[48] T. S. Da Silva, M. S. Silveira, and F. Maurer, "Ten lessons learned from integrating interaction design and Agile development," Proceedings AGILE 2013, pp. 42-49, 2013.

[49] K. Kuusinen and T. Mikkonen, "Designing User Experience for Mobile Apps: Long-Term Product Owner Perspective," 2013 20th Asia-Pacific Software Engineering Conference (APSEC), vol. 1, pp. 535-540, 2013.

[50] B. Losada, M. Urretavizcaya, and I. Fernández-Castro, "A guide to agile development of interactive software with a "User Objectives"-driven methodology." Science of Computer Programming, vol. 78, no. 11, pp. 2268-2281, nov 2013.
[51] J. Mcginn and A. Ramirez Chang, "RITE + Krug : A Combination of Usability Test Methods for Agile Design," Journal of Usability Studies, vol. 8, no. 3, pp. 61-68, 2013.

[52] S. Prior, A. Waller, R. Black, and T. Kroll, "Use of an agile bridge in the development of assistive technology," Proceedings of the SIGCHI Conference on Human Factors in Computing System, pp. 1579-1588, 2013.

[53] K. A. Abdelouhab, D. Idoughi, and C. Kolski, "Agile \& user centric SOA based service design framework applied in disaster management," 2014 1st International Conference on Information and Communication Technologies for Disaster Management, ICT-DM 2014, 2014.

[54] S. M. Butt, O. Azura, M. M. B, N. T. Inam, and M. Butt, "Incorporation of Usability Evaluation Methods in Agile Software Model," na, pp. 193199, 2014

[55] A. Gordillo, E. Barra, S. Aguirre, and J. Quemada, "The usefulness of usability and user experience evaluation methods on an eLearning platform development from a developer's perspective: A case study," 2014 IEEE Frontiers in Education Conference (FIE) Proceedings, vol. 2015-Febru, no. February, pp. 1-8, 2014. [Online]. Available: http://www.scopus.com/inward/record.url?eid=2s2.0-84938150347\&partnerID=tZOtx3y1

[56] G. Jurca, T. D. Hellmann, and F. Maurer, "Integrating agile and usercentered design: A systematic mapping and review of evaluation and validation studies of agile-UX," Proceedings - 2014 Agile Conference, AGILE 2014, pp. 24-32, 2014.

[57] E. Kropp and K. Koischwitz, "User-centered-design in agile RE through an On-site User Experience Consultant," 2014 IEEE 2nd International Workshop on Usability and Accessibility Focused Requirements Engineering, UsARE 2014 - Proceedings, pp. 9-12, 2014.

[58] W. A. Rahim, W. M. Isa, A. M. Lokman, N. F. Taharim, and N. D. Wahid, "Engineering m-learning using agile user-centered design," Proceedings - 2014 8th International Conference on Next Generation Mobile Applications, Services and Technologies, NGMAST 2014, pp. 60-65, 2014.

[59] S. Anwar, Y. H. Motla, Y. Siddiq, S. Asghar, M. S. Hassan, and Z. I. Khan, "User-centered design practices in serum development process: A distinctive advantage?" 17th IEEE International Multi Topic Conference: Collaborative and Sustainable Development of Technologies, IEEE INMIC 2014 - Proceedings, pp. 161-166, 2015.

[60] J. K. Blomkvist, J. Persson, and J. Åberg, "Communication through Boundary Objects in Distributed Agile Teams," Proceedings of the 33rd Annual ACM Conference on Human Factors in Computing Systems CHI '15, pp. 1875-1884, 2015.

[61] T. S. Da Silva, F. F. Silveira, M. S. Silveira, T. Hellmann, and F. Maurer, "A systematic mapping on agile UCD across the major agile and HCI conferences," Lecture Notes in Computer Science (including subseries Lecture Notes in Artificial Intelligence and Lecture Notes in Bioinformatics), vol. 9159, pp. 86-100, 2015.

[62] L. A. Rojas and J. A. Macías, "An Agile Information-ArchitectureDriven Approach for the Development of User-Centered Interactive Software," Proceedings of the XVI International Conference on Human Computer Interaction - Interacción '15, pp. 1-8, 2015. [Online]. Available: http://dl.acm.org/citation.cfm?id=2829875.2829919

[63] D. Salah, R. Paige, and P. Cairns, "Patterns for integrating agile development processes and user centred design," ... of the 20th European Conference on ..., pp. 1-10, 2015. [Online]. Available: http://dl.acm.org/citation.cfm?id=2855341

[64] T. Ovad and L. B. Larsen, "The Prevalence of UX Design in Agile Development Processes in Industry," 2015 Agile Conference, pp. 4049, 2015.

[65] A. Y. Wale-Kolade, "Integrating usability work into a large interorganisational agile development project: Tactics developed by usability designers." Journal of Systems \& Software, pp. 54-66, feb 2015.

[66] T. Pfeiffer, J. Hellmers, E. M. Sch??n, and J. Thomaschewski, "Empowering User Interfaces for Industrie 4.0," Proceedings of the IEEE, vol 104, no. 5, pp. 986-996, 2016.

[67] G. Meszaros and J. Aston, "Adding usability testing to an agile project," in Proceedings of the conference on AGILE 2006. Washington, DC, USA: IEEE Computer Society, 2006, pp. 289-294.

[68] J. Ungar and J. White, "Agile user centered design: enter the design studio - a case study," in CHI '08 extended abstracts on Human factors in computing systems, ser. CHI EA '08. New York, NY, USA: ACM, 2008, pp. 2167-2178. 
[69] M. Singh, "U-scrum: An agile methodology for promoting usability," in Proceedings of the Agile 2008. Washington, DC, USA: IEEE Computer Society, 2008, pp. 555-560.

[70] Z. Hussain, M. Lechner, H. Milchrahm, S. Shahzad, W. Slany, M. Umgeher, T. Vlk, and P. Wolkerstorfer, "User interface design for a mobile multimedia application: An iterative approach," in Proceedings of the First International Conference on Advances in Computer-Human Interaction, ser. ACHI '08. Washington, DC, USA: IEEE Computer Society, 2008, pp. 189-194. 